\title{
electrostatic charge on intraocular lenses
}

\begin{abstract}
Purpose An electronic meter that measures electrostatic charge was designed and built. The hypothesis tested was that an intraocular lens (IOL) attains a charge during handling in the operating theatre and that washing the IOL with balanced salt solution (BSS) would result in a reduction in the surface charge. Once exposed to the air a charged IOL may attract particulate matter to its surface before implantation into the eye.

Methods This experiment was performed in the operating theatre under sterile conditions mimicking cataract surgery. The lens box was opened onto a trolley and the lens container opened by a scrubbed assistant. The operator (also scrubbed) removed the lens with MacPherson's forceps. The charge on the lens

This study was designed to determine whether manipulation of an IOL in the operating theatre prior to implantation resulted in a measurable electrostatic charge on the surface of the lens. A literature search provided only one reference on the subject, where the use of a piezoelectric gun to remove charge was recommended but no measurement of the magnitude of the surface charge was made. ${ }^{2}$

The rationale behind washing the IOL before implantation is that the surface charge consists of excess surface electrons or holes (a 'hole' being the absence of an electron). If these are placed in contact with a conducting electrolytic solution then they can be bound with free ions in the solution and thus removed from the lens surface, resulting in a reduction of the total surface charge.
\end{abstract} was then measured without delay, or after the lens had been washed with 1-2 $\mathrm{ml}$ of BSS. Results The mean charge on unwashed lenses was $1.43 \times 10^{-10} \mathrm{C}(n=12)$ and on washed lenses was $0.59 \times 10^{-10} \mathrm{C}(n=10)$. The difference in the charges was significant $(p=0.03$, unpaired $t$-test).

Conclusion Washing of an IOL prior to implantation will reduce the surface charge and is therefore also likely to reduce the chance of contamination.

Key words Cataract, Charge, Electrostatic, Implantation, IOL, Washing

This study was initiated after a conversation in the operating theatre on whether washing of an intraocular lens (IOL) prior to implantation in the eye was necessary or not. The materials used in lens manufacture are non-conducting and have dielectric properties. This would allow them to accumulate an electrostatic charge during handling. Any charged object exposed to the atmosphere will attract particulate matter due to the induction or presence of an opposite charge in the particle. The air in an operating theatre contains dust, desquamated skin scales and airborne bacteria. ${ }^{1}$ Introduction of these into the eye could contribute to post-operative uveitis or at worst cause bacterial endophthalmitis.

\section{Charge measurement}

\section{Principle of charge measurement}

Electrostatic charge can be collected on the plates of a capacitor. The stored charge generates a voltage given by the relationship $Q=C V$, where $Q$ is the charge in coulombs, $C$ the capacitance in farads and $V$ the voltage in volts. ${ }^{3}$ An electronic device for measuring charge was designed, based on this principle.

\section{Details of charge meter}

The charge meter used a $1 \mu \mathrm{F}$ polypropylene capacitor to collect electrostatic charge. One side of the capacitor was connected to earth, the other to an electrically conducting plate. This formed the charge collecting plate of the meter. This side of the capacitor was also connected to the input of an amplifier. Amplification was achieved using a two-stage DC inverting amplifier based on high-quality, low-noise operational amplifiers. The first stage had a gain of around unity, but a very high input resistance of $10 \mathrm{M} \Omega$. This gave the circuit an RC constant of 10 (input resistance $\times$ capacitance); the charge stored on the capacitor thus took $10 \mathrm{~s}$ to decay to approximately one-third of its initial value (as $Q=Q_{0} \mathrm{e}^{-t / R C}$ ), facilitating measurement of the voltage. The second stage of the amplifier had a lower input resistance but a much higher gain, variable to a maximum of 100 .
N.P. Davies

Imperial College (Biophysics)

Prince Consort Road

South Kensington

London SW7 2BZ, UK

Tel: +44 (0)171 594 7674/2

Fax: +44 (0)171 5890191

S.R. Rassam

Western Eye Hospital

St Mary's Hospital NHS Trust

London, UK

S.M. Shah

St Bartholomew's Hospital

The Royal Hospitals NHS

Trust

London, UK 


\section{Calibration}

The calibration system used a resistor divider network to reduce the supply voltage of the meter by a known amount (factors of $10^{-4}, 10^{-5}$ or $10^{-6}$ ). The following procedure was used to calibrate the meter: The supply voltage was measured and thus the output voltage of the resistor divider circuit calculated. The output of the amplifier was measured with the known small voltage from the divider at its input. The gain of the amplifier was then calculated from the ratio of the output voltage to the input voltage. This calibration was repeated during the course of the experiment to ensure that the gain of the device was not drifting. The output was measured using a digital multimeter. The unit containing the circuit and control panel was electrically isolated to avoid any other electrical interference.

\section{Experimental method}

The experiment was performed in the operating theatre. The experimenters were scrubbed and gowned, wearing sterile cotton gowns and antistatic microsurgical gloves. An instrument trolley was covered in a sterile cotton drape. A $2 \mathrm{ml}$ syringe, a gallipot, a lacrimal cannula and a pair of MacPherson's forceps were opened on the trolley. The gallipot was filled with balanced salt solution (BSS).

One of the authors acted as the surgeon, whilst another acted as the assistant. The charge meter was placed on the operating table in the position of the patient's head. It was zeroed and calibrated. Each author touched the charge plate and no charge was detected on either. The assistant opened the lenses, one at a time. The outer wrapper was discarded, the inner cardboard box opened and the lens, in its plastic sterile container was dropped onto the trolley. The lens container was opened. The lens was removed by the surgeon (using the MacPherson's forceps) and placed onto the charge plate without delay. Alternatively, the lens was washed by the assistant before charge measurement. Washing was performed either by filling the well holding the lens with BSS prior to removal (the 'immersed' group), or by washing the lens directly after removal from the container (the 'rinsed' group), 1-2 $\mathrm{ml}$ of BSS from a $2 \mathrm{ml}$ syringe and lacrimal cannula being used in both cases. After washing, the lens was immediately placed onto the charge plate. The output of the meter was documented in each case. Calibration was rechecked during the course of the experiment to allow for small variations in the gain of the amplifier.

\section{Lenses used}

Expired lenses were used for the study. None of these had been previously opened or damaged in any way. The lenses used were of varying power, type and manufacturer: one-piece PMMA lenses, three-piece PMMA lenses (prolene haptics), one foldable silicone lens and one heparin surface modified (HSM) lens. The lens powers varied from $+15 \mathrm{D}$ to $+27 \mathrm{D}$. As the charge measurement removes charge from the lens surface onto the plate of the capacitor, it was not possible to test each individual lens both unwashed and then washed. The two groups of lenses did not, therefore, form matched pairs.

\section{Results}

The results are presented in Tables 1-3. The gain of the meter for each calibration performed during the experiment is given in Table 1 . There was some variation in the gain of the device during the time course of the experiment. However, this variation is automatically accounted for in the charge calculation, as seen below. The charge on the lenses was calculated using the formula:

$$
Q=V_{0} \cdot C / G
$$

where $Q$ is the charge in coulombs, $C$ is $1 \times 10^{-6}$ farads, $G$ is the gain calculated from calibration of the meter and $V_{0}$ its measured output in volts.

The data were analysed for mean, standard deviation, normal distribution and statistical significance.

Examination of the data showed that all values were approximately normally distributed. The modulus of the measured charge was used in the calculations. Either polarity will induce a charge of the opposite sign on nearby non-conducting objects resulting in an attractive force towards the IOL, and thus the magnitude of the charge is the key factor in determining whether particulate matter will be attracted towards the lens surface. The mean charge for the unwashed group $(n=12)$ was $1.43 \times 10^{-10} \mathrm{C}\left(\mathrm{SD} 1.19 \times 10^{-10}\right)$ and for the washed group $(n=10)$ was $0.59 \times 10^{-10} \mathrm{C}$ $\left(\mathrm{SD} 0.67 \times 10^{-110} \mathrm{C}\right)$. As the lenses were not matched pairs and the modulus of the charge measured was used, a one-tailed unpaired Student's $t$-test was performed $(p=0.03)$. The same test was also performed on the meter output voltage $(p=0.03)$ and thus variation in the gain of the meter is seen to be unimportant. The charge measured on the silicone foldable lens and the HSM lens was of the same order of magnitude as the charge on the PMMA lenses (Table 2). The washed lenses were divided into two subgroups: rinsed and immersed. The mean charge of the rinsed group was $0.43 \times 10^{-10} \mathrm{C}$ $\left(\mathrm{SD} 0.48 \times 10^{-10} \mathrm{C}\right)$ and of the immersed group $0.75 \times 10^{-10} \mathrm{C}\left(\mathrm{SD} 0.84 \times 10^{-10} \mathrm{C}\right)(p=0.26)$. Comparison of these two subgroups with the unwashed lenses was significant $(p=0.01)$ for the rinsed group but not significant $(p=0.10)$ for the immersed group. These statistics are summarised in Table 3.

Table 1. Gain of amplifier

\begin{tabular}{ll}
\hline Lens number & Gain \\
\hline Unwashed lenses $(1-6)$ & 106.4 \\
Unwashed lenses $(7-12)$ & 117.7 \\
Washed lenses $(1-10)$ & 109.9 \\
\hline
\end{tabular}


Table 2. Eleectrostatic charge on washed and unwashed intraocular lenses

\begin{tabular}{|c|c|c|c|c|c|c|}
\hline \multicolumn{3}{|c|}{ Unwashed lenses } & \multicolumn{4}{|c|}{ Washed lenses } \\
\hline$\overline{V_{0}(\mathrm{mV})}$ & $Q\left(C \times 10^{-10}\right)$ & Type & $V_{0}(\mathrm{mV})$ & $Q\left(\mathrm{C} \times 10^{-10}\right)$ & Type & Method \\
\hline-11.6 & -1.090 & PMMA one-piece & -6.1 & -0.555 & PMMA one-piece & Immersed \\
\hline+30.1 & +2.829 & PMMA one-piece & +23.9 & +2.175 & PMMA one-piece & Immersed \\
\hline+16.7 & +1.570 & PMMA one-piece & -1.4 & -0.127 & PMMA one-piece & Immersed \\
\hline+13.2 & +1.241 & PMMA one-piece & +3.5 & +0.123 & PMMA one-piece & Immersed \\
\hline+4.3 & +0.404 & PMMA one-piece & -8.4 & -0.765 & PMMA one-piece & Immersed \\
\hline+30.9 & +3.473 & PMMA one-piece & +2.0 & +0.182 & PMMA three-piece & Rinsed \\
\hline+0.2 & +0.017 & PMMA one-piece & +13.3 & +1.210 & PMMA three-piece & Rinsed \\
\hline+30.9 & +2.624 & PMMA one-piece & +0.5 & +0.046 & PMMA three-piece & Rinsed \\
\hline+27.6 & +2.594 & PMMA three-piece & +6.3 & +0.573 & PMMA three-piece & Rinsed \\
\hline-3.4 & -0.289 & PMMA three-piece & -11.5 & -0.977 & PMMA one-piece & Rinsed \\
\hline-11.5 & -0.977 & Silicone foldable & & & & \\
\hline+0.2 & +0.017 & PMMA HSM & & & & \\
\hline
\end{tabular}

PMMA, polymethylmethacrylate; HSM, heparin surface modified.

\section{Discussion}

The incidence of bacterial endophthalmitis is fortunately low, figures varying from $0.07 \%$ to $0.1 \%$. ${ }^{4,5}$ Studies of anterior chamber aspirates at the end of surgery show high bacterial contamination rates, varying from $5 \%$ to $43 \%{ }^{6-10}$

The incidence of inflammatory response after cataract surgery has been measured as $5.5 \%^{11}$ and in one study fibrinous membrane formation on the surface of the IOL occurred in $7.6 \%$ of cases. ${ }^{12}$ One potential source of dust particles or micro-organisms entering the eye is the surface of the IOL. Shah and Spalton have shown that it is possible to study lens surface cytology in vivo with a specular microscope ${ }^{13}$ and have identified two distinct responses: an early small cell response and a later giant cell response. ${ }^{14}$ It may be that the cellular response on the IOL surface is due, in part, to surface contaminants that were present on the lens after manufacture or that alighted on its surface whilst the lens was manipulated during surgery. Interestingly, the giant cell response is significantly reduced in HSM lenses. ${ }^{15}$ It was suggested that this might be due to either enhanced biocompatibility or reduced inflammatory cell adhesion

Table 3. Sumary of results

\begin{tabular}{lc}
\hline Unwashed lenses & \\
Mean charge & $1.43 \times 10^{-10} \mathrm{C}$ \\
SD & $1.19 \times 10^{-10} \mathrm{C}$ \\
Washed lenses & \\
All & \\
Mean charge & $0.59 \times 10^{-10} \mathrm{C}$ \\
SD & $0.67 \times 10^{-10} \mathrm{C}$ \\
Rinsed & \\
$\quad$ Mean charge & $0.43 \times 10^{-10} \mathrm{C}$ \\
SD & $0.48 \times 10^{-10} \mathrm{C}$ \\
Immersed & \\
$\quad$ Mean charge & $0.75 \times 10^{-10} \mathrm{C}$ \\
SD & $0.84 \times 10^{-10} \mathrm{C}$ \\
Unpaired t-test & \\
Unwashed vs all washed & $p=0.03$ \\
Unwashed vs rinsed & $p=0.01$ \\
Unwashed vs immersed & $p=0.10$ \\
Rinsed vs immersed & $p=0.26$ \\
\hline
\end{tabular}

to the surface of the lens. It has been shown that bacterial adhesion to HSM lenses is reduced ${ }^{16}$ and this may also be a factor.

Vafidis et al. ${ }^{17}$ recorded a bacterial contamination rate of $26 \%$ on dry IOLs placed on the external eye during cataract surgery (for an exposure duration similar to that of the implanted lens) and of $15 \%$ on IOLs exposed solely to the operating theatre air. Conjunctival irrigation yielded a contamination rate of $8 \%$ and swabbing the fornix only $6 \%$. It appears from these figures that PMMA has a propensity to collect bacterial contaminants. Vafidis et al. proposed that opening of the IOL should be delayed until the last possible moment and that contact with the external eye should be minimised, although no assessment of the effects of washing the IOL with BSS was made. They also suggested that electrostatic charge on the surface of the IOL might be a contributory factor in the high rate of bacterial contamination seen.

In this study we provide the first quantification of electrostatic charge on IOLs after handling in the operating theatre, under conditions mimicking cataract surgery. This charge is of the order of $10^{-10} \mathrm{C}$ and is equal to the charge on approximately $10^{9}$ electrons. We have also demonstrated that washing the IOL with BSS reduces the charge on its surface. The results indicate that both methods of washing reduce surface charge, although a greater effect was seen in the rinsed group $(p=0.01)$ than the immersed group $(p=0.1)$. Although rinsing the IOL with BSS may appear to be more effective than immersing it, we feel it is difficult to comment on any specific advantage of one method of washing over another, as the number of IOLs in each subgroup is very small. It is unlikely that further charge could be induced whilst removing the IOL from its container, as once its surface is wet with a conducting electrolytic solution any free electrons or holes would be rapidly bound with free ions.

The ratio of the means of the charge measured in the two groups is 2.5. As electrostatic force is proportional to the product of the charge on two bodies $\left(F \propto q_{1} q_{2} / r^{2}\right)$, this factor indicates a mean reduction of attractive force on nearby particles of a factor of 6.25 . Washing the IOL with BSS before implantation would therefore be 
advantageous over implantation without washing. To capitalise on the reduction of surface charge the IOL should be washed as soon as possible after opening the container, as once it is wet no further static charge will be accumulated.

In conclusion, washing an IOL with BSS prior to implantation has the advantage of reducing the likelihood of surface contamination, due to a reduction in electrostatic charge and a concomitant reduction of any attractive force on nearby airborne particles. This is achieved with minimal additional time for the procedure, no extra cost and no increased risk to the patient.

\section{References}

1. Howorth FH. Prevention of airborne infection during surgery. Lancet 1985;I:386-8.

2. Tennant JL, Smirmaul H, Meltzer DW. Use of the piezoelectric gun to remove surface charge from intraocular lenses. J Am Int Ocul Implant Soc 1984;10:486-7.

3. Horowitz P, Hill W. The art of electronics. 2nd ed. Cambridge: Cambridge University Press, 1989:20-22.

4. Kattan HM, Flynn HW Jr, Pflugfelder SC, Robertson C, Forster RK. Nosocomial endophthalmitis survey: current incidence of infection after intraocular surgery. Ophthalmology 1991;98:227-38.

5. Weinberg RS (Chair). Basic and clinical science course section 9. San Francisco: American Academy of Ophthalmology, 1994-5:100-8.

6. Samad A, Solomon LD, Miller RA, Mendelson J. Anterior chamber contamination after uncomplicated phacoemulsification and intraocular lens implantation. Am J Ophthalmol 1995;120:143-50.
7. Egger SF, Huber-Spitzy V, Schold C, Schneider B, Grabne Bacterial contamination during extracapsular cataract extraction: prospective study on 200 consecutive cases. Ophthalmologica 1994;208:77-81.

8. Manners TD, Chitkara DK, Marsh PJ, Stoddart MG. Anter chamber aspirate cultures after small incision cataract surgery. Br J Ophthalmol 1995;79:878-80.

9. Sherwood DR, Rich WJ, Jacob JS, Hart RJ, Fairchild YL. Bacterial contamination of intraocular and extraocular fluid during extracapsular cataract extraction. Eye 1989;3:308-12

10. Dickey JB, Thompson KD, Jay WM. Anterior chamber aspirate cultures after uncomplicated cataract surgery. Am Ophthalmol 1991;112:278-82.

11. Drolsum L, Davanger M, Haaskjold E. Risk factors for an inflammatory response after extracapsular cataract extraction and posterior chamber IOL. Acta Ophthalmol (Copenh) 1994;72:21-6.

12. Nishi O. Fibrinous membrane formation on the posterior chamber lens during the early postoperative period. J Cataract Refract Surg 1988;14:73-7.

13. Shah SM, Spalton DJ, Muir MK. Specular microscopy of the anterior intraocular lens surface. Eye 1993;7:707-10.

14. Shah SM, Spalton DJ. Natural history of cellular deposits on the anterior intraocular lens surface. J Cataract Refract Surg 1995;21:466-71.

15. Shah SM, Spalton DJ. Comparison of the postoperative inflammatory response in the normal eye with heparinsurface-modified and poly(methyl methacrylate) intraocular lenses. J Cataract Refract Surg 1995;21:579-85.

16. Portoles M, Refojo MF, Leong FL. Reduced bacterial adhesion to heparin-surface-modified intraocular lenses. J Cataract Refract Surg 1993;19:755-9.

17. Vafidis GC, Marsh RJ, Stacey AR. Bacterial contamination of intraocular lens surgery. Br J Ophthalmol 1984;68:520-3. 